\title{
Influence of Chronic UV Exposure and Lifestyle on Facial Skin Photo-Aging --- Results from a Pilot Study
}

\author{
Suminori Akiba ${ }^{1}$, Reiko Shinkura ${ }^{1}$, Kukizo Miyamoto ${ }^{2}$, Greg Hillebrand ${ }^{2}$, Naohito Yamaguchi ${ }^{3}$, \\ and Masamitsu Ichihashi ${ }^{4}$
}

In order to better understand the effect of chronic sun exposure on facial skin photo-aging and to identify the factors affecting it, we planned a study in two areas in Japan, Akita and Kagoshima, which correspond to the low and high sun exposure environments, respectively. As a first step, we conducted a pilot study in the two areas, examining 195 subjects. Hyperpigmentation and wrinkling were measured with a high-resolution digital video imaging system. As expected, people in Kagoshima had darker skin, higher visual grades of facial hyperpigmentation, and more facial wrinkles than people in Akita, reflecting the difference of UV exposure levels in the two areas. Both the grades of hyper-pigmentation and number of wrinkles increased in a roughly linear fashion with the advancement of age. On the other hand, the effect of gender was different in those two skin photo-aging parameters. Women had higher hyperpigmentation grades $(P=0.012)$ and less wrinkles $(P=0.004)$ than men. Interestingly, postmenopausal women had higher grades of hyper-pigmentation than pre-menopausal women. Neither sun exposure index for darkness nor wrinkling showed any significant differences by menopausal status. In this pilot study, we collected information on various factors, including lifestyles. The results of detail analysis will be presented elsewhere. In the present analysis, we found that the grade of hyper-pigmentation was not related to total hours spent outside in life but was affected by various factors, including toe-nail zinc levels. On the other hand, the number of wrinkles was significantly related to total hours spent outside in life.

The most important risk factors of non-melanoma skin cancer are chronic sun exposure, age and male sex. All of them are strongly related to higher levels of UV exposure. The present study confirmed that chronic sun exposure, age and male sex were strong risk factors of the wrinkle number. The number of wrinkles was significantly related to total hours of sun exposure in life, increased in a roughly linear fashion with the advancement of age, and was larger in men than in women. In epidemiological studies of UV-related skin cancer, the number of wrinkles, which can be easily measured with a high-resolution digital video imaging system as shown in this study, may be a good marker of total sun exposure in life. J Jpidemiol, 1999 ; 9 : S136-S142.

UV, lifestyles, skin, photo-aging

Chronic sun exposure causes various forms of skin damage. While the qualitative aspects of skin damage caused by chronic sun exposure are well-known, few studies have been conducted to quantitatively understand the relationship between chronic sun exposure and premature skin aging ${ }^{1)}$. In order to better understand the effect of chronic sun exposure on skin photoaging and to identify the factors affecting it, we planned a study in two areas in Japan, Akita and Kagoshima, which corresponded to the low and high sun exposure environments, respectively. As a first step, we conducted a pilot study, examining 195 subjects in the two areas. In this paper, we will report the results of preliminary analysis using the data obtained from the pilot study.

${ }^{1}$ Faculty of Medicine, Kagoshima University.

${ }^{2}$ The Procter \& Gamble Company.

${ }^{3}$ Cancer Information and Epidemiology Division, National Cancer Center Research Institute.

${ }^{4}$ Kobe University School of Medicine.

Address for correspondence : Suminori Akiba, Sakuragaoka 8-35-1, Faculty of Medicine, Kagoshima University. 


\section{SUBJECTS AND METHODS}

Subjects: Study subjects were healthy males and females in their 20s and 50s who lived in Akita or Kagoshima for their entire life. We tried to examine twenty-five subjects for each stratum created by gender, age group, and study location. In total, 200 office workers in NTT and its related companies were invited to participate in this study. Some of the study subjects were workers' family members. Those who were pregnant and in ill-health were excluded from the study subjects.

Skin color tone: Skin color tone was measured on the middle of the forehead (sun-exposed site) and upper inner arm (non sun-exposed site) using a Minolta Chromameter Model 300, which was calibrated against standard color plates supplied with the instrument. The measurement provided three indices of skin color tones, i.e., $\mathrm{L}^{*}, \mathrm{a}^{*}$, and $\mathrm{b}^{*}$, which represents the lightness, redness, and yellowness of skin, respectively. Three measurements were obtained from the forehead and upper inner arm. The average of these three measurements in each skin site was used in further analysis. Since the skin color of the forehead represents facultative plus constitutive skin color whereas the skin color of the upper inner arm represents primarily constitutive skin color, J Lock-Anderson et al. ${ }^{2)}$ proposed to calculate Sun Exposure Index (SEI) using the following formula:

$\mathrm{SEI}=[(\mathrm{E}-\mathrm{U}) / \mathrm{U}] * 100$,

where $E$ and $U$ were skin color in the sun-exposed and sununexposed skin sites, respectively. In the present study, the SEI for darkness was calculated using the following analogous formula:

SEID $=-[(\mathrm{Lf}-\mathrm{La}) / \mathrm{La}]^{*} 100$, where $\mathrm{Lf}$ and La were the $\mathrm{L}^{*}$ values (lightness) of skin in the forehead and upper inner arm. respectively. As SEID increases, facultative skin darkness increases relative to constitutive skin color.

Hyper-pigmented spot area visual grading: A 5-point grading scale for the severity of hyper-pigmentation was developed using images captured with the Facial Color Image Capturing System, FACIS98. FACIS98 uses a high-resolution 3CCD digital camera equipped with a strobe flash lighting for quantifying facial skin color tone and hyper-pigmented spots. Three judges viewed each subject's facial image and assigned a grade(1-5, 5 being most severe) using the standard images as reference. The mean score of the 3 judges for each subject was used in data analysis. The mean coefficient of variance between judges' score was $15 \%$.

Wrinkles: The count and total length of wrinkles in the region of the upper cheek, and temporal areas next to the eyes were objectively measured using a computer-aided Wrinkle Imaging System, WIS, which identifies a wrinkle, a facial line, greater than $5 \mathrm{~mm}$ in length and $0.16 \mathrm{~mm}$ in width. In WIS, a high quality $3 \mathrm{CCD}$ video camera captures the left and right side images of the facial area to be examined and the captured facial images are converted into a digital form by a PC-based image processing system. Facial illumination was provided by a quartz tungsten halogen light source in order to enhance skin appearance features, including wrinkles and texture. In the present study, we presented the results of analysis using the data of wrinkle numbers only since the wrinkle number and total length of wrinkles showed quite similar results.

Questionnaire: Interview of a subject was conducted at the examination site using a structured questionnaire. Part I of the questionnaire, which was administered via face-to-face interview by a trained physician, contained the questions regarding medical history, including OB-GYN and skin health history. Part II of the questionnaire, administered by two trained interviewers, was to collect information on the subject's sun exposure history, life styles including smoking, drinking, and dietary habits.

Toenail samples: Toenail clippings were obtained from each subject and analyzed using ICP-MS for $\mathrm{Zn}, \mathrm{Se}, \mathrm{Mg}, \mathrm{Mn}, \mathrm{AS}$, and other trace elements.

Statistical analysis: Regression analysis was conducted where the values of various skin parameters were regressed on age, study site, and other factors, including life-style related variables. All $P$ values presented are two-sided.

\section{RESULT}

Our surveys were conducted in Kagoshima and Akita in the first and second week of April, 1998, respectively. We invited 200 subjects to participate in the study. Since 5 subjects did not show up for various reasons, the total number of study subjects in this pilot study was 195 . The age and sex specific distributions of study subjects in Akita and Kagoshima are shown in Table 1. In the analysis of sun exposure index for darkness (SEID), the following three points were noted as expected: people in Kagoshima had darker facial skin than people in Akita; older people had darker skin than younger people; and men had darker skin than women (Table 1). The effects of area, age and gender on SEID were highly significant $(P<0.001)$ in statistical analysis using a linear regression model incorporating area, age, and gender. Table 1 also shows the results of hyper-pigmentation and wrinkling. The subjects in Kagoshima had higher grades of hyper-pigmentation and more wrinkles than in Akita; older people had higher grades of hyper-pigmentation and more wrinkles. The effects of area and age on the hyper-pigmentation grade and wrinkle numbers were highly significant in regression analysis $(P<0.001)$.

In regression analysis where SEID was regressed on age, the constant term of the regression model, corresponding to the SEID value at birth, was positive and statistically significantly different from zero (Table 2). In the regression analysis of hyper-pigmentation, the constant term was small but it was 
Table 1. Sun Exposure Index for Darkness.

\begin{tabular}{|c|c|c|c|c|c|c|c|c|}
\hline & \multicolumn{4}{|c|}{$\begin{array}{c}\text { Female } \\
\text { age }\end{array}$} & \multicolumn{4}{|c|}{$\begin{array}{c}\text { Male } \\
\text { age }\end{array}$} \\
\hline & $20-29$ & & $50-59$ & & $20-29$ & & $50-59$ & \\
\hline & Mean & SD & Mean & SD & Mean & SD & Mean & $\mathrm{SD}$ \\
\hline \multicolumn{9}{|l|}{$\mathrm{n}$} \\
\hline Akita & 25 & & 25 & & 24 & & 24 & \\
\hline Kagoshima & 24 & & 25 & & 24 & & 24 & \\
\hline \multicolumn{9}{|l|}{ SEID $(\%)$} \\
\hline Akita & 4.9 & 3.9 & 6.4 & 3.4 & 9.3 & 3.3 & 14.1 & 3.9 \\
\hline Kagoshima & 10.9 & 4.9 & 13.6 & 3.9 & 16.5 & 5.4 & 16.9 & 3.6 \\
\hline \multicolumn{9}{|l|}{ Spot grade } \\
\hline Akita & 1.65 & 0.62 & 3.11 & 0.89 & 1.39 & 0.39 & 2.67 & 0.59 \\
\hline Kagoshima & 2.13 & 0.71 & 3.61 & 0.86 & 1.69 & 0.83 & 2.78 & 0.85 \\
\hline \multicolumn{9}{|c|}{ Wrinkle number } \\
\hline Akita & 6.4 & 7.0 & 19.3 & 9.3 & 7.6 & 5.4 & 28.1 & 9.4 \\
\hline Kagoshima & 9.3 & 7.3 & 30.5 & 12.1 & 13.1 & 6.3 & 33.8 & 10.9 \\
\hline
\end{tabular}

Table 2. Results of regression analysis.

\begin{tabular}{|c|c|c|c|c|}
\hline \multicolumn{5}{|c|}{ dependent variable : SEID (\%) } \\
\hline \multirow{2}{*}{$\begin{array}{l}\text { independent variables } \\
\text { in men }\end{array}$} & \multicolumn{2}{|c|}{ Kagoshima } & \multicolumn{2}{|c|}{ Akita } \\
\hline & Estimate & SE. & Estimate & SE. \\
\hline Constant & 0.16 & 0.02 & 0.06 & 0.01 \\
\hline Age & 0.00014 & 0.00043 & 0.00155 & 0.00034 \\
\hline \multicolumn{5}{|l|}{ in women } \\
\hline Constant & 0.08 & 0.02 & 0.04 & 0.02 \\
\hline Age & 0.00120 & 0.00056 & 0.00052 & 0.00054 \\
\hline \multicolumn{5}{|c|}{ dependent variable : spot grade } \\
\hline \multirow{2}{*}{$\begin{array}{l}\text { independent variables } \\
\text { in men }\end{array}$} & \multicolumn{2}{|c|}{ Kagoshima } & \multicolumn{2}{|c|}{ Akita } \\
\hline & Estimate & SE. & Estimate & SE. \\
\hline Constant & 0.80 & 0.31 & 0.43 & 0.19 \\
\hline Age & 0.038 & 0.008 & 0.042 & 0.005 \\
\hline \multicolumn{5}{|l|}{ in women } \\
\hline Constant & 1.38 & 0.31 & 0.65 & 0.36 \\
\hline Age & 0.033 & 0.009 & 0.041 & 0.011 \\
\hline \multicolumn{5}{|c|}{ dependent variable : wrinkle number } \\
\hline \multirow{2}{*}{$\begin{array}{l}\text { independent variables } \\
\text { in men }\end{array}$} & \multicolumn{2}{|c|}{ Kagoshima } & \multicolumn{2}{|c|}{ Akita } \\
\hline & Estimate & SE. & Estimate & SE. \\
\hline Constant & -2.60 & 3.30 & -7.6 & 3.0 \\
\hline Age & 0.69 & 0.08 & 0.67 & 0.07 \\
\hline \multicolumn{5}{|l|}{ in women } \\
\hline Constant & -5.38 & 4.27 & -4.96 & 3.93 \\
\hline Age & 0.65 & 0.12 & 0.46 & 0.12 \\
\hline
\end{tabular}

The estimate of a regression coefficient and SE were obtained from multiple regression analysis. In women, the effect of menopause ( $=1$ for menopausal women, $=0$ otherwise) was adjusted in the regression analysis. 
also statistically significantly larger than zero. On the other hand, the regression analysis of wrinkle numbers found the constant term to be not significantly different from zero.

Since the UV exposure level in Kagoshima was much higher than that in Akita, we expected that the regression coefficient of age was larger in Kagoshima than that in Akita in the regression analysis of SEID, hyper-pigmentation grades and wrinkle numbers. As shown in Table 2, the regression coefficients of age in the analysis of SEID were different in the two areas and in the two sexes whereas those in the analyses of hyper-pigmentation and wrinkle numbers were not much different in the two areas or in both sexes. Note here, however, that the standard errors of SEID's coeficient were large when compared to those of hyperpigemenatation grades and wrinkle numbers.

The effect of gender on skin photo-aging was different in the hyper-pigmentation grade and wrinkle numbers. Women had higer grades of hyper-pigmentation $(P=0.012)$ but less wrinkles $(P=0.004)$ than men. Since sex hormones were known to affect skin photo-aging, we examined the effect of menopause on skin parameters in the women aged 50 or over (Table 3). As shown in Table 3, post-menopausal women had higher grades of hyper-pigmented spots than pre-menopausal women. On the other hand, neither SEID nor wrinkling showed any significant differences by menopausal status.

We calculated total hours spent outside for life based on the information obtained from our questionnaire survey. In this calculation, the use of hat and so on was not taken into account. Total hours spent outside was positively associated with wrinkle numbers $(P=0.005)$ whereas neither SEID nor spots grades had a statistically significant association with total hours spent outside.

We collected toenail samples and measured concentration of various trace elements in this pilot study. Since skin aging is suspected to be related to free radical activity, the present analysis put a focus on zinc and selenium, which are structural components of SOD (superoxidase dismutase) and GST (Glutathione-S-Transferase), respectively, the enzymes having antioxidant activity. The concentration of zinc in toenail samples showed an inverse correlation with the grade of hyper-pigmented spots in females(Table 4). Even among men, a similar result was obtained when the subjects were restricted to nonsmokers although the association was not statistically significant. Smoking itself did not show any statistically significant associations with SEID, wrinkling, or hyper-pigmentation grades. Neither associated was toenail selenium levels.

\section{DISCUSSION}

The present study showed that the people in Kagoshima had darker skin color, higher grades of hyper-pigmentation and more wrinkles than the people in Akita, reflecting the stronger UV exposure in Kagoshima than in Akita. The annual UVB radiation falling on Kagoshima was estimated to be about 1.5 times that of Akita ${ }^{3}$. Our study results are in general agreement with that of the report by Masano et al., who measured skin color lightness in various locations in Japan and found that Japanese living in sunny Okinawa had skin approximately $6 \mathrm{~L}$ units darker than people living in less sunny Akita prefecture ${ }^{4}$.

In the regression analysis where SEID was regressed on age,

Table 3. effect of menopause on skin aging parameters.

\begin{tabular}{|c|c|c|c|c|c|}
\hline \multirow{2}{*}{ skin parameters } & \multicolumn{2}{|c|}{ Premenopause } & \multicolumn{2}{|c|}{ Postmenopause } & \multirow{2}{*}{$P$ value } \\
\hline & mean & $\mathrm{SD}$ & mean & SD & \\
\hline SEID (\%) & 11.8 & 5.7 & 9.0 & 4.6 & 0.368 \\
\hline Spot grade & 2.98 & 0.98 & 3.57 & 0.79 & 0.014 \\
\hline Wrinkle number & 24.8 & 12.6 & 24.9 & 12.0 & 0.694 \\
\hline
\end{tabular}

Table 4. Toe nail zinc concentration and skin parameters in women.

\begin{tabular}{lcccccccc}
\hline \multirow{2}{*}{ skin parameter } & \multicolumn{8}{c}{ zinc level } \\
& \multicolumn{2}{c}{ low } & \multicolumn{2}{c}{ medium } & \multicolumn{2}{c}{ high } & P for trend \\
& mean* & SE & mean & SE & mean & SE & \\
\hline SEID (\%) & 9.1 & 0.9 & 8.5 & 0.9 & 9.1 & 0.7 & 0.713 \\
spot grade & 2.9 & 0.2 & 2.7 & 0.2 & 2.6 & 0.1 & 0.037 \\
wrinkle number & 15.0 & 2.0 & 18.3 & 1.9 & 17.3 & 1.5 & 0.991 \\
\hline
\end{tabular}

* The mean, its SE and $\mathrm{P}$ for trend were obtained from a regression model adjusting for study site, age and menopausal status. 
the constant term in the regression model, corresponding to the value of SEID at age zero, was larger than zero. In other words, the extrapolation of the SEID data obtained from the survey of subjects in their 20s and 50s to age zero resulted in the SEID value larger than zero at birth. Our results support the studies reporting that the forehead was already more pigmented than the medial arm area even in the newborn infant ${ }^{5}$. Another possibility is the age specific curve of SEID is convex rather than linear as shown in Fig 1. This problem will be addressed by the main study, which will examine a wider range age than the pilot study.

The hyper-pigmentation grade increased in a linear fashion with the advancement of age, as shown in Fig 2. However, here again, the constant term in the regression analysis was slightly larger than zero. The mechanisms involved in the increasing severity of hyper-pigmentation with the advancement of age is not well understood. Although the number of active melanocytes are known to decrease with the advancement of age after age 30, the hyper-pigmentation in older persons are more severe than in younger persons as was shown in many studies including the present one ${ }^{5}$.

The regression analysis where the wrinkle number was regressed on age did not give evidence that the constant term was statistically significantly different from zero. The wrinkle number increased in a roughly linear fashion with the advancement of age as shown in Fig 3. It should be noted here that our analysis included all the wrinkles identified by WIS, which can identify all the facial lines greater than $5 \mathrm{~mm}$ in length and $0.16 \mathrm{~mm}$ in width. However, the UV-induced wrinkling is known to be deeper and wider than the wrinkling associated with chronological aging ${ }^{1,6,7}$. Therefore, some exploratory analysis is warranted to make a distinction between the wrinkling caused by chronological aging using several different criteria of a wrinkle other than what was used in the present study.

Although men had more wrinkles than women, menopause did not affect wrinkle numbers in women. The sex difference in the wrinkle number may be largely reflecting the sex difference in sun exposure levels rather than hormonal environment. Indeed, men had larger SEID values than women. Our finding

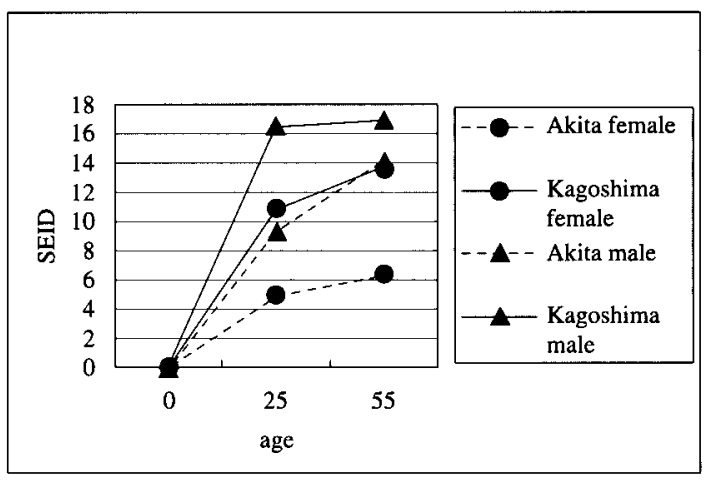

Figure 1. Age specific curve of SEID \%.

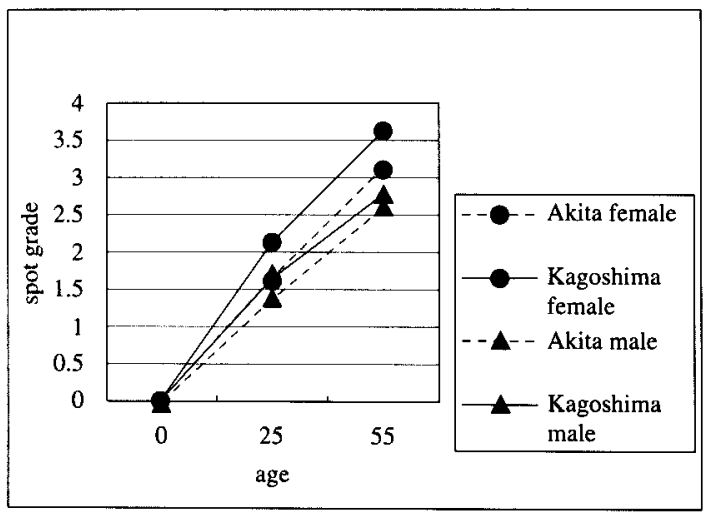

Figure 2. Age specific curve of hyperpigmentation grade. 


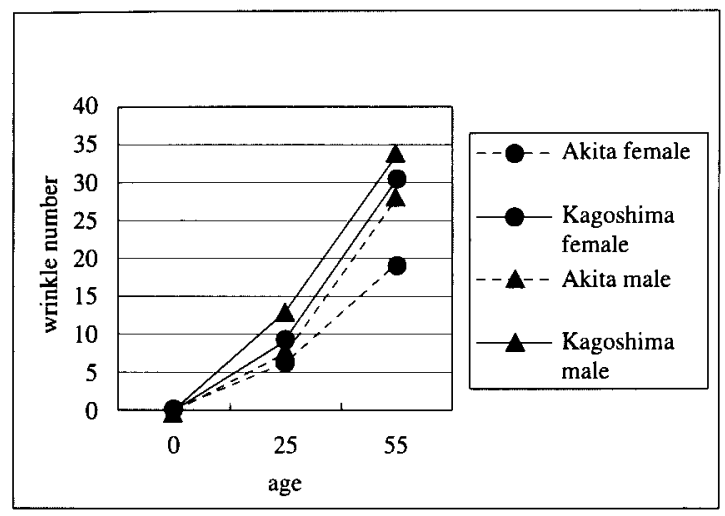

Figure 3. Age specific curve of wrinkle number.

confirmed the results obtained from the dermatological component of the first National Health and Nutrition Examination Survey, 1971-74, in the US, where men showed more frequent skin damage than women ${ }^{8}$. In the present study, women had higher grades of hyper-pigmentation than men, suggesting that hyper-pigmentation grades were strongly affected by the factors other than chronic sun exposure. Arai et al reported that women had more pigmentation than men in the youth through middle ages whereas the gender effect was reversed in the older ages ${ }^{9}$. It is of interest to know whether our main study, which will include people older than age 60 , confirm their findings. Another interesting finding relevant to the sex difference is the effect of menopause. Menopause resulted in an increase, rather than a decrease, of hyper-pigmentation. Further investigations are necessary to elucidate the mechanisms involved in the aggravation of hyper-pigmentation after menopause.

In retrospective epidemiological studies, efforts have been made to estimate life-time sun exposure using information on recreational, occupational and other types of exposure to sunlight obtained from questionnaire surveys. However, the information thus obtained is a subjective measure and its reliability is doubtful. J Andersen-Lock et al reported that the SEI reflected life time UV exposure ${ }^{2}$. Their argument was not supported in the present study, where SEID was not related to the total hours spent outside in life. The wrinkled skin is reported to be a good indicator of life-time UV exposure. L Fritschi et al. reported that the severity of wrinkles examined with silicone-rubber casts reflects life-time UV exposure when their grading system was used ${ }^{10)}$. The present study showed that the number of wrinkles measured with a high-resolution digital video imaging system was useful in quantifying the magnitude of wrinkling.

One of our interesting findings was the inverse relationship between the zinc concentration of toenail and the grade of hyper-pigmented spots. At this stage of analysis, we can not totally deny the possibility that the observed association was a result of the bias caused by the factors that were not controlled in our preliminary analysis. However, it should be noted here that the effects of zinc on skin photo-aging and pigmentation were reported by many studies. In the skin, UVB light (wavelength range of $280-320 \mathrm{~nm}$ ) is mainly absorbed by the epidermis, where the continual turnover of its basic cellular unit, the keratinocyte, takes place. UVB irradiation was reported to produce apoptotic keratinocytes called sunburn cells. The application of zinc chloride prior to UV exposure was reported to reduce the number of sunburn cells ${ }^{11)}$. On the other hand, UVA (320-400 nm) penetrates the epidermis more deeply than UVB and generates free radicals, which frequently cause irreversible damages. Zinc is known to be a structural element of copper-zinc-SOD, which may act as the scavenger of active oxygen. Pretreatment of skin with SOD is reported to protect against PUVA-induced inflammatory reactions not only in murine, but also in human skin ${ }^{12}$.

The most important risk factors of non-melanoma skin cancer are chronic sun exposure, age and male sex ${ }^{13}$. All of them are strongly related to higher levels of UV exposure. The present study showed that chronic sun exposure, age and male sex are strong risk factors of the wrinkle number. The number of wrinkles was significantly related to total hours of sun exposure in life, increased in a roughly linear fashion with the advancement of age, and was larger in men than in women. In epidemiological studies of UV-related skin cancer, the number of wrinkles may be a good marker of total sun exposure in life. The results obtained from this pilot study warranted the conduct of the full fledged main study.

\section{REFERENCE}

1. Lavker RM. Cutaneous aging: Chronic versus photoaging. In; Gilchrest BA, ed. Photoaging. Blackwell Science, 1995:123-135. 
2. Lock-Andersen J, Knudstorp ND, Wulf HC. Facultative skin pigmentation in Caucasians: an objective biological indicator of lifetime exposure to ultraviolet radiation? Brit J Dermatology,1988;138:826-832.

3. Takahashi K, Kawana K, Okubo T. Estimation of regional UV-B levels as exposure indices for ecological and environmental epidemiologic studies. JUOEH, 1996;18:51-60.

4. Masano M, Muramatsu Y, Yuasa S. Measurement of optical properties of the skin by developped methods and its application. J Soc Cosmet Chem Japan,1982;16:1518.

5. Post Pw, Krauss AN, Waldman S et al. Skin reflectance of newborn infants from 25 to 44 weeks gestational age. Human Biology 1976;48:541-547.

6. Bernstein EF, Chen YQ, Kopp JB et al. Long term sun exposure alters the collagen of the papillary dermis: comparison of sun-protected and photo-aged skin by Northern analysis, immunohistochemical staining, and confocal laser scanning microscopy. J Am Acad Dermatol, 1996;34: 209-218.

7. Fisher GJ, Wang ZQ, Datta SC et al. Pathophysiology of premature skin aging induced by ultraviolet light. New Engl J Med 1997;337:1419-1428.

8. Engel A, Johnson ML, Haynes SG. Health effects of sunlight exposure in the United States. Arch Dermatol, 1988;124:72-79.

9. Arai S, Hara Y, Mimura K, et al.Analysis of pigmentation in human skin.Quantification of ultraviolet ray images by digital-image-processing. JSCCJ, 1989;23:31-42.

10. Fritschi L, Battistutta D, Strutton GM, Green A. A noninvasive measure of photoageing. Int $\mathbf{J}$ Epidemiol, 1995;24:150-154.

11. Record IR, Jannes M, Dreosti IE. Protection of zinc against UVA and UVB-induced cellular and genomic damage in vivo and in vitro. Biol Trace Elem Res 1996; 53:19-25.

12. Filipe $\mathbf{P}$, Emerit I, Vassy $\mathrm{J}$ et al. Epidermal localization and protective effects of topically applied superoxidase dismutase. Exp Dermatol,1997;6:116-121.

13. Scotto J, Fears TR, Kraemer KH, Et al. Nonmelanoma skin cancer. In; Schottenfeld D and Fraumeni JR, eds. Cancer Epidemiology and Prevention. $2^{\text {nd }}$ ed. Oxford University Press, 1996;1313-1330. 